\title{
Post-prandial worsening of angina: All due to changes in cardiac output?
}

\author{
A J Cowley, L J Fullwood, K Stainer, E Harrison, A F Muller, J R Hampton
}

\begin{abstract}
Background-The precise mechanism leading to the post-prandial worsening of angina has yet to be adequately defined. It has been attributed to an increase in double product but is perhaps more likely to be related to an increase in cardiac output after food. This study was designed to evaluate the effects of food on patients' exercise tolerance and compare these with changes in haemodynamic variables.

Methods-23 patients with chronic stable angina who had post-prandial worsening of their angina were studied. The patients were evaluated on two occasions and at each visit they underwent two symptom limited treadmill exercise tests. They remained fasting on the first visit and were given a $1400 \mathrm{kcal}$ meal 60 minutes before the second exercise test on the second visit. Time to onset of $1 \mathrm{~mm}$ ST segment depression, heart rate, systemic arterial blood pressure, and cardiac output were measured at rest and during exercise.
\end{abstract}

Results-There were no differences in any of the variables during the two exercise tests on the day the patients remained fasting. After the meal exercise tolerance fell significantly by 136 seconds and the stage at which $1 \mathrm{~mm}$ ST segment depression was first seen was also significantly reduced. Resting cardiac output increased significantly by $0.861 / \mathrm{min}$ with the patients sitting and by $0.891 / \mathrm{min}$ standing. The exercise times after food were significantly related to cardiac output even when fasting times were taken into account. Resting heart rate increased significantly by 8.3 beats per minute sitting and 10.4 beats per minute standing. There was little change in blood pressure and no evidence that the double product predicted the post-prandial exercise time.

Conclusions-Worsening of angina was related to the increase in cardiac output after a meal and successful treatment will depend upon the prevention of this increase.

The observation that eating can worsen angina was first made over two centuries ago. ${ }^{1}$ However, it has been surprisingly difficult to demonstrate the mechanism by which this occurs. Intuitively it might be expected that a meal causes an increase in cardiac output and that this accounts for the worsening of symptoms, but it has been almost universally impossible to show this with invasive techniques and in most studies there has been no consistent increase in cardiac output postprandially. ${ }^{23}$ Post-prandial deterioration in angina has therefore been attributed to an increase in heart rate and blood pressure which is taken to indicate an increase in myocardial oxygen demand. ${ }^{4}$ Even the consistency of these changes is in doubt because simple non-invasive measurements showed that food causes a reduction in blood pressure and little if any increase in heart rate in elderly subjects, who are those most likely to have angina. ${ }^{5}$ The difficulty in defining adequately the mechanism of post-prandial worsening of angina therefore seems to be attributable to the invasive nature of the techniques used.

We showed that cardiac output can be measured non-invasively and reliably by analysis of respiratory gases ${ }^{6}$ and that a modest meal increased the cardiac output of a group of normal subjects. ${ }^{7}$ If methodological problems are the reason for the difficulties in showing the cause of post-prandial angina then use of a non-invasive technique may overcome these. The purpose of this study was to evaluate quantitatively the effect of food on the exercise tolerance of patients with angina and to try to determine the relevance of haemodynamic changes to any deterioration in angina.

\section{Patients and methods}

Twenty three patients with chronic stable angina participated in the study. All described a worsening of their angina after meals. Twenty one were men and the patients were aged 46-69 years. All were limited by angina on treadmill exercise testing. Antianginal medication was stopped, in those patients taking prophylactic treatment, at least 24 hours before each exercise test except for sublingual glyceryl trinitrate which was permitted up to two hours before treadmill exercise testing.

All patients were fully familiarised with the study protocol on at least two occasions before entering the study. They were asked to attend the exercise laboratory at 11 am on two days one week apart having fasted from midnight. On each visit they underwent two symptom 
limited exercise tests separated by at least 120 minutes. On the first (control) day the patients remained fasting and after the first test on the second (test) day they were given a standard meal of $1400 \mathrm{kcal}$ to be eaten 60 minutes before the second test.

\section{TREADMILL EXERCISE TESTS}

All patients exercised to a symptom limited maximum with a modified low level Bruce protocol. The speed and slope of the treadmill were increased after three minutes at each of the following stages:

$\begin{array}{cllllll} & I & I I & I I I & I V & V & V I \\ \text { Speed } & & & & & & \\ (\mathrm{km} / \mathrm{h}) & 2 \cdot 7 & 2 \cdot 7 & 2 \cdot 7 & 2 \cdot 7 & 4.0 & 5.4 \\ \text { Slope }\left({ }^{\circ}\right) & 0 & 1.3 & 2.6 & 4.3 & 5.4 & 6.3\end{array}$

The exercise stages were prolonged to four minutes during stages I and III to enable cardiac output to be measured.

Heart rate was recorded at rest and at the end of each stage of exercise. Blood pressure was measured by auscultation with a sphygmomanometer at rest and at the end of each stage of exercise. Standard 12 lead electrocardiograms were recorded at rest and every three minutes during the treadmill test and at the end of exercise.

Time to onset of $1 \mathrm{~mm} \mathrm{ST} \mathrm{segment} \mathrm{depres-}$ sion in any lead from the 12 lead electrocardiogram was measured in those patients who had changes in all four tests.

\section{CARDIAC OUTPUT}

We measured cardiac output by an indirect Fick principle by monitoring respiratory gases with a mass spectrometer (V G Medicals). Carbon dioxide was used as the indicator. Carbon dioxide production was calculated from minute ventilation and mixed expired carbon dioxide concentration, the partial pressure of carbon dioxide in pulmonary venous (systemic arterial) blood was derived from end tidal carbon dioxide concentration, and the partial pressure in mixed venous blood was measured after a rebreathing manoeuvre. These three variables were used to solve the Fick equation.

Cardiac output was measured with the patients sitting, standing, and at fixed submaximal workloads at the end of stages I and III during the treadmill exercise test. The coefficient of variation of the method was $8 \cdot 2 \%$.

\section{STATISTICAL ANALYSIS}

The effect of visit (week 1 compared with week 2) and exercise test (first compared with second) on maximal exercise duration and each of the haemodynamic measurements was assessed by a three factor analysis of variance with patient identity included as a blocking factor. ${ }^{8}$ We used the statistical modelling package $G L I M .^{9}$ In each case the magnitude of the variable associated with the first order interaction term involving the factors visit and exercise, gives a measure of the effect of the meal.

The time to onset of $1 \mathrm{~mm} \mathrm{ST} \mathrm{segment}$ depression was coded according to the stage at which it first occurred. Patients who stopped exercising without this degree of ST depression developing were coded as stage VII-that is, beyond the end of exercise. The within visit difference in score (between the second and first exercise test) was calculated to give a measure of the effect of repeated exercise (visit 1 ) and the additional effect of the meal (visit 2). The difference between the visit differences can therefore be interpreted as a measure of the effect of the meal. We examined these with a Wilcoxon signed rank test.

The relation between cardiac output and maximal exercise time was investigated by multiple regression techniques. Specifically, a regression model was fitted that related exercise time after the meal to the exercise time before the meal. Change in cardiac output was then added to this regression to see whether this additional information improved the prediction of exercise time after the meal.

\section{Results}

All the patients had moderate angina and when they were studied they were not taking medication. Three patients were unable to complete stage I of the protocol on at least one occasion and seven could not complete stage II for all four tests.

\section{SYMPTOM LIMITED EXERCISE TOLERANCE}

Figure 1 shows the exercise tolerance of the patients on the control and study days. On the
Figure 1 Individual and mean (SEM) values for the symptom limited exercise tolerance of the patients on the control and test days $(p<0.01$ on the test day).

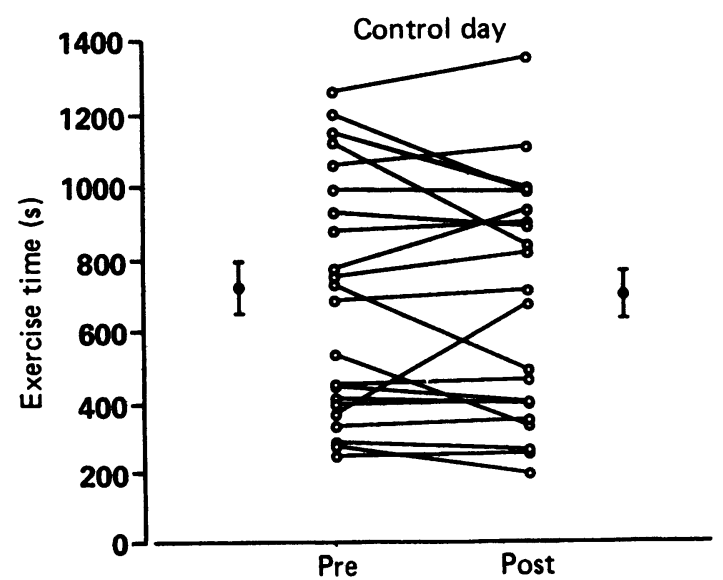


Figure 2 Individual and mean (SEM) values for time to onset of $1 \mathrm{~mm} S T$ segment depression in those patients in whom this occurred on the control and test days $(p<0.05$ on the test day).

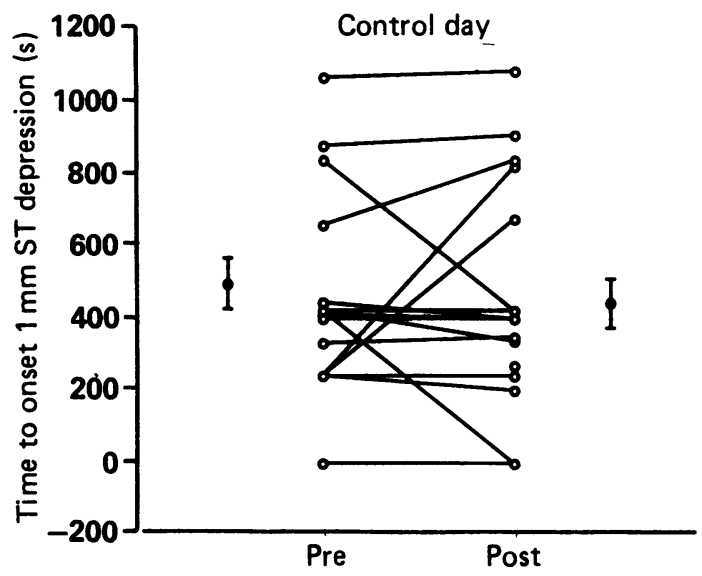

control day 11 patients had greater exercise times on the second exercise test compared with only three patients on the test day. The mean (SEM) times for the control day were 725 (70) seconds for the first test and 703 (69) seconds for the second test. On the test day the times were $783(65)$ and 626 (59) seconds. Therefore on the control day there was a small decrease in exercise time for the second test which may indicate the effects of repeated testing to a symptom limited maximum. When this was taken into account with analysis of variance the effect of the meal was to reduce exercise time by 136 seconds $(p<0.01)$.

\section{ST SEGMENT CHANGES}

Figure 2 shows the time to onset of $1 \mathrm{~mm} \mathrm{ST}$ segment depression in those patients who developed it. On the control day $1 \mathrm{~mm} \mathrm{ST}$ segment depression occurred earlier in the second exercise test in five patients and at the same stage in 11 patients. On the test day it was seen earlier in 15 patients during the second test and at the same time in five patients $(\mathrm{p}<0.05)$.

\section{HEART RATE AND BLOOD PRESSURE}

Table 1 shows the mean (SEM) values of heart rate with the patients sitting, standing, and at the end of exercise. Model based estimates indicated that heart rate was eight beats per minute higher after the meal when the patients

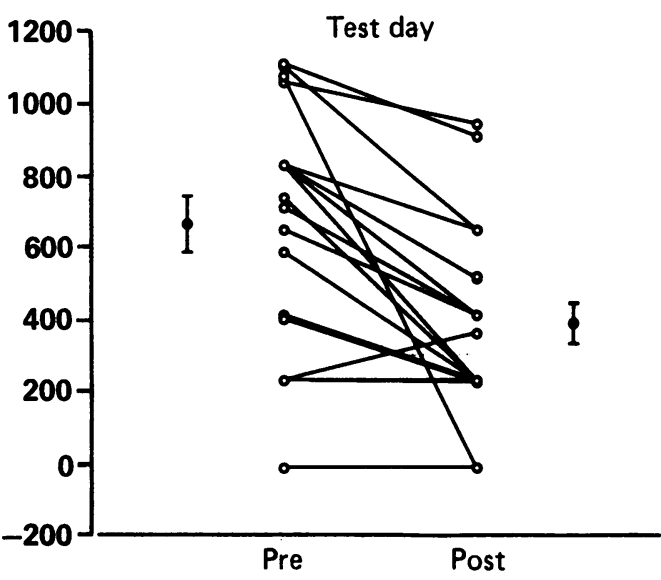

were sitting ( $p<0.05)$ and 10 beats per minute when they were standing $(p<0.01)$.

There was no significant effect of the meal on systolic or diastolic blood pressure at any time. The double product (mean arterial blood pressure $\times$ heart rate) measured with the patients sitting was 6329 (381) and 6228 (457) on the control day and 6277 (323) and 6844 (530) on the test day. These values were not significantly different and the changes in double product were not related to exercise time after the meal.

\section{CARDIAC OUTPUT}

All patients were capable of performing the respiratory manoeuvres needed for cardiac output to be calculated. Table 2 shows the values of cardiac output with the patients sitting, standing, and the last measurement obtained during the exercise test. The variable number of patients who completed a measurement during exercise are also shown. Model based calculations show that the meal increased cardiac output by $0.86 \mathrm{l} / \mathrm{min}$ with the patients sitting $(\mathrm{p}<0.01)$ and by $0.89 \mathrm{l} / \mathrm{min}(\mathrm{p}<0.01)$ with them standing. The effect of the meal, however, was not significant at or near peak exercise.

The increase in resting measurements of cardiac output in response to the meal was a useful predictor of the decrease in exercise after the meal. Adding the change in cardiac output to a regression model relating post-meal exer-

Table 1 Mean (SEM) values of heart rate (beats/minute) with the patients sitting, standing, and at the end of exercise

\begin{tabular}{|c|c|c|c|c|c|c|}
\hline & \multicolumn{2}{|l|}{ Control day } & \multicolumn{2}{|l|}{ Test day } & \multirow{2}{*}{$\begin{array}{l}\text { Change } \\
\text { in control } \\
\text { post - pre }\end{array}$} & \multirow{2}{*}{$\begin{array}{l}\text { Model based } \\
\text { effect of } \\
\text { meal }\end{array}$} \\
\hline & Pre & Post & Pre & Post & & \\
\hline $\begin{array}{l}\text { Sitting } \\
\text { Standing } \\
\text { End point }\end{array}$ & $\begin{array}{r}66.9(2.6) \\
75.7(2.7) \\
106.9(5.5)\end{array}$ & $\begin{array}{r}65.1(2.4) \\
74.9(2.6) \\
107.2(5.7)\end{array}$ & $\begin{array}{r}64 \cdot 8(2 \cdot 3) \\
72 \cdot 7(2 \cdot 5) \\
111 \cdot 6(5 \cdot 0)\end{array}$ & $\begin{array}{r}71 \cdot 1(3.4) \\
82.4(3.0) \\
109.9(5.5)\end{array}$ & $\begin{array}{r}-0.94 \\
-0.78 \\
0.19\end{array}$ & $\begin{array}{c}8 \cdot 34^{\star} \\
10 \cdot 43 \dagger \\
-2.00\end{array}$ \\
\hline
\end{tabular}

${ }^{\star} \mathrm{p}<0.02,+\mathrm{p}<0.01$.

Table 2 Mean (SEM) values of cardiac output with the patients sitting, standing, and the last value obtained from those in whom measurements were made during exercise

\begin{tabular}{|c|c|c|c|c|c|c|}
\hline & \multicolumn{2}{|c|}{ Control day } & \multicolumn{2}{|l|}{ Test day } & \multirow{2}{*}{$\begin{array}{l}\text { Change } \\
\text { in control } \\
\text { post - pre }\end{array}$} & \multirow{2}{*}{$\begin{array}{l}\text { Model based } \\
\text { effect of } \\
\text { meal }\end{array}$} \\
\hline & Pre & Post & Pre & Post & & \\
\hline $\begin{array}{l}\text { Sitting } \\
\text { Standing } \\
\text { End point } \\
\text { Number at end point }\end{array}$ & $\begin{array}{l}4 \cdot 0(0 \cdot 2) \\
3 \cdot 8(2 \cdot 7) \\
8 \cdot 3(0 \cdot 5) \\
18\end{array}$ & $\begin{array}{r}3.9(0.2) \\
3.9(2.6) \\
7.9(0.5) \\
21\end{array}$ & $\begin{array}{c}4.0(0 \cdot 2) \\
3 \cdot 9(2 \cdot 5) \\
8 \cdot 4(0 \cdot 5) \\
16\end{array}$ & $\begin{array}{l}4.8(0 \cdot 2) \\
4 \cdot 8(3 \cdot 0) \\
8 \cdot 8(0 \cdot 4) \\
20\end{array}$ & $\begin{array}{r}-0.02 \\
0.03 \\
-0.44\end{array}$ & $\begin{array}{l}0 \cdot 86^{\star} \\
0 \cdot 89^{\star} \\
1.01\end{array}$ \\
\hline
\end{tabular}


cise time to pre-meal time significantly improved the predictive accuracy $(p=0.039)$.

\section{Discussion}

In this study we showed that the exercise tolerance of patients with angina can be reduced quite substantially by a modest meal. We also showed that this reduction in exercise tolerance is related to the increase in cardiac output in response to the meal.

The fact that a meal may worsen the symptoms of some patients with angina is clinically well recognised ${ }^{10}$ but it has been surprisingly difficult to demonstrate with any consistency the mechanism. It has been known for many years that a meal increases cardiac output ${ }^{11}$ but more recent attempts to confirm the original observation have often been unsuccessful. ${ }^{23}$ In part this is likely to be because the invasive techniques used in themselves can cause changes in haemodynamic function and thereby mask the expected post-prandial increase in cardiac output. Non-invasive techniques have proved more reliable in showing the changes in cardiac output after food. ${ }^{12}$ In normal volunteers we showed that a modest meal may increase resting cardiac output by up to $30 \%{ }^{7}$ The present study showed a similar increase in cardiac output at rest which was maintained and was additive to the changes during submaximal exercise. Furthermore, there was a relation between the changes in cardiac output and the change in exercise tolerance of the patients such that those patients who had the greatest increase in cardiac output had the greatest reduction in exercise tolerance.

In the absence of demonstrable changes in cardiac output the post-prandial worsening of angina has previously been ascribed to an increased myocardial oxygen consumption shown by an increase in the product of heart rate and blood pressure. ${ }^{4}$ Although there is evidence that patients develop angina within a fairly narrow range of double product for each individual ${ }^{4}$ the effect of food may be paradoxically to lower the double product by a reduction in blood pressure. In our study we saw the expected increase in heart rate but little change in either systolic or diastolic blood pressure and little change in the double product. The factors that determine myocardial oxygen consumption are complex and the combination of blood pressure and heart rate can only be an approximation. ${ }^{1314}$ The pumping action of the myocardium is of obvious importance because this must relate directly to energy consumption and therefore oxygen utilisation; but until now, reliable non-invasive measurements of cardiac output have not been possible. It is therefore not surprising that the increase in cardiac output we showed in this study is more closely related to the reduction in patients' symptom limited exercise tolerance than changes in double product.
The factors responsible for the increase in cardiac output are unclear. Digestion is a metabolically active process that is associated with an increase in systemic oxygen consumption. Gut blood flow is increased ${ }^{15}$ and this seems to be met by an increase in cardiac output. Redirection of blood from other vascular beds does not contribute to the enhanced blood supply to the gut. Part of the physiological stimulus for the increased cardiac output is increased activity of the sympathetic nervous system and this has implications for the treatment of post-prandial angina. ${ }^{16}$ Drugs, such as $\beta$ blockers, may prevent the increase in cardiac output with obvious benefit. An alternative way of treating patients would be with agents that prevent the increase in gut blood flow or alternatively redirect blood from other tissues and obviate the need for an increase in cardiac output. Patients may find that taking smaller meals more frequently is helpful.

A meal is an important cause of worsening of patients' angina. Its effects are related to the magnitude of the increase in cardiac output and treatment will depend upon the prevention of this increase.

1 Heberden W. Some account of a disorder of the breast. Medical Transactions of the College of Physicians of London 1772;2:59-67.

2 Goldstein RE, Redwood DR, Rosing DR, Beiser GD, Epstein SE. Alterations in the circulatory response to exercise following a meal and their relationship to postprandial angina pectoris. Circulation 1971;44:90-100.

3 Jones WB, Thomas HD, Reeves TJ. Circulatory and ones WB, Thomas HD, Reeves TJ. Circulatory and
ventilatory responses to postprandial exercise. Am Heart J ventilatory respons

4 Robinson BF. Relation of heart rate and systolic blood pressure to the onset of pain in angina pectoris. Circulation pressure to the on

5 Potter JF, Heseltine D, Hartley G, Matthews J, Macdonald IA, James OFW. Effects of meal composition on the postprandial blood pressure, catecholamine and insulin changes in the elderly. Clin Sci 1989;77:265-72.

6 Cowley AJ, Stainer K, Murphy DT, Murphy J, Hampton JR. A non invasive method for measuring cardiac output: the effect of Christmas lunch. Lancet 1986;ii:1422-3.

7 Yi JJ, Fullwood L, Stainer K, Cowley AJ, Hampton JR. Effects of food on the central and peripheral haemodynamic response to upright exercise in normal volunteers. $\mathrm{Br}$ Heart J 1990;63:22-5.

8 Armitage P, Berry G. Statistical methods in medical research. 2nd ed. Oxford: Blackwell Scientific Publications, 1987.

9 Payne CD, ed. The GLIM system release 3.77, revision a. Oxford: Numerical Alograthims Group, 1986.

10 Wayne EJ, Graybiel A. Observations on the effect of food, gastric distension, external temperature and repeated gastric distension, external temperature and repeated exercise on angina of effort, wi
dolore. Clin Sci 1934;1:287-304.

11 Grollman A. Physiological variations in the cardiac output of Grollman A. Physiological variations in
man. Am J Physiol 1929;89:366-70.

12 Kelbæk H, Munck O, Christensen NJ, Godtfredsen J. Central haemodynamic changes after a meal. Br Heart $J$ 1989;61:506-9.

13 Sarnoff SJ, Braunwald E, Welch GH, Case RB, Stainsby WN, Macruz R. Hemodynamic determinants of oxygen consumption of the heart with special reference to the tension-time index. Am J Physiol 1958;192:148-56.

14 Braunwald E, Sarnoff SJ, Case RB, Stainsby WN, Welch GH. Hemodynamic determinants of coronary flow: effect of changes in aortic pressure and cardiac output on the relationship between myocardial oxygen consumption and coronary flow. Am J Physiol 1958;192:157-63.

15 Qamar MI, Read AE, Skidmore R, Evans JM, Wells PNT Transcutaneous doppler ultrasound measurement of superior mesenteric artery blood flow in man. Gut 1986; 27:100-5.

16 Kelbaek H, Munck O, Christensen NJ, Godtfredsen J. Autonomic nervous control of postprandial hemodynamic
changes at rest and upright exercise. $J$ Appl Physiol 1987;63:1862-5. 